\title{
ZUR KRITIK UND ERKLÄRUNG EINIGER LIEDER WALTHERS VON DER VOGELWEIDE.
}

43, 9 ff. (L.) Durch die untersuchungen Braunes, Beitr. $42,130 \mathrm{f}$. scheint mir erwiesen, daß in der 3. strophe dieses gedichtes von den frauen in der 3. person gesprochen wird. Aber der echte wortlaut der verse 30. 31 ist, wie ich glaube, noch nicht gefunden. Braune will lesen: (Wir wellen daz diu stoetekeit) den guoten wîbcn gar ein krône sî. kunnen si mit zühten sin gemeit... Ich komme nicht über den zweisilbigen auftakt hinweg, und auch zu der annahme von kunnens mit versetzter betonung ${ }^{1}$ ) würde ich mich nur im äußersten notfall entschließen. Vor allem aber halte ich es, bevor man eine andere sichere stelle nachgewiesen hat, für unmöglich, daß ein krône absolut gebraucht 'das höchste' bedeuten könne. Mein sprachgefühl stimmt hier mit dem des schreibers a überein; seine conjectur ob allin guotin dingin ist freilich unrichtig.

Gewöhnlich bezeichnet die durch einen genitiv oder eine präpositionalformel gegebene bestimmung von krône die gattung, von der etwas das höchste darstellt, z. b. bei Walther selbst 107, 29 gelêrter fürsten krône; bei Hartmann MF. 215, 29 si was von kinde und muoz mê sîn mîn krône denjenigen, für den etwas subjectiv das höchste ist. Bei Morungen MF. 133, 29. 30 hat krône eine zweifache bestimmung: diu mînes herzen ein wunne und ein krôn ist vor allen frouwen diech noch hân gesehen. Wollte man unsere Waltherstelle nach analogie des Hartmannschen verses beurteilen und zugeben, daß die bestimmung von krône auch im einfachen dativ stehen könne, so käme der sinn heraus: 'wir sind der ansicht, daß für die

2) Vgl. Wilmanns, Einleitung zur Waltherausgabe s. 40.44.

Beitrkge zur geschichle der deutschen oprache. 4. 
frauen subjectiv, in ihrer schätzung, beständigkeit das höchste sei'. Das ist aber natürlich nicht gemeint; die männer glauben, daß beständigkeit objectiv die höchste weibliche tugend ist. Die richtige lesung ist nach meiner überzeugung der gïete an wîbe(n) gar ein krône sî. Durch die klammer drücke ich aus, daß ich es vorläufig unbestimmt lassen will, ob wîp im singular oder im plural stand.

In jedem fall konnte der text von den abschreibern leicht mißverstanden werden, wenn man annimmt, daß die elision des - $e$ von gïete bezeichnet war, und bedenkt, daß in alten handschriften $u_{0}$ und $\ddot{i} e$ nicht streng geschieden werden. Stand in der vorlage der gut an wibe(n), so konnte dies sehr leicht als der guoten wîbe aufgefaßt werden.

Dieses primäre mißverständnis liegt noch vor in der guoten frauwen $\mathrm{E}$. Die nächstverwandte handschrift $\mathrm{F}$ hat den artikel eingebüßt. Wenn auch y (nach Braunes stammbaum) den artikel verloren hatte, so stand da git an wibe(n). Alle abkömmlinge von $\mathrm{y}$ haben giot für das adjectiv gehalten. $\mathrm{s}$ hat an noch als präposition erkannt und deshalb an guoten wiben conjiciert, ${ }^{*} \mathrm{BC}$ dagegen git an wie ${ }^{*} \mathrm{EF}$ für guoten gehalten und seiner tendenz entsprechend iu vorgesetzt.

Jetzt gilt es noch, die wahl zwischen wîbe und wîben zu treffen. Ich entscheide mich für wîbe. Daß die lesart $\mathrm{E}$ dazu vorzüglich stimmt, bedarf keiner ausführung. Aber auch in den anderen handschriften konnte sehr leicht der plural für den immerhin etwas seltneren generellen artikellosen singular eintreten. Bei $\mathrm{F}$ und ${ }^{*} \mathrm{BC}$ kommt hinzu, dab die endung -en von guoten (wibe) auf den plural hinzuweisen schien. Für den singular spricht, daß in der 4. strophe von den männern in der einzahl gesprochen wird. Setzt man wîbe ein, so kann man v. 31 mit E kan si schreiben, und der metrische anstoß ist behoben. Natürlich ist dann auch v. 36 wîbe zu lesen.

Die auf kan si mit zühten sîn gemeit folgende zeile 32 sô stêt diu lilje wol der rôsen bî wị̂d in den commentaren nicht richtig erklärt. Bei Pfeiffer heißt es: 'die verbíndung der lilie mit der rose gebrauchen die mhd. dichter, Walther voran, öfter zur bildlichen bezeichnung des höchsten inbegriffs körperlicher sowohl als sittlicher reize'. Er verweist auf die 
stellen (nach Lachmanns zählung) 74, 31. 53, 38. 28, 7. Und Wilmanns findet an eben diesen stellen die verbindung von lilie und rose als bild leiblicher schönheit.

Nun, von leiblicher schönheit ist an unserer stelle gewiß nicht die rede, und dann muß doch auch erklärt werden, warum hier, wo es sich um einen sittlichen, oder saggen wir lieber gesellschaftlichen vorzug handelt, gerade lilie und rose genannt werden. Es ist nicht Walthers art, mit den worten über die dinge bloß hinwegzustreifen ${ }^{1}$ ). Er will nicht sagen: 'versteht eine frau es, mit anstand heiter zu sein, so ist der höchste inbegriff von sittlichen oder gesellschaftlichen reizen da'; das kann er schon deshalb nicht, weil er eben die stcetekeit für die höchste weibliche tugend erklärt hat. Der sinn ist vielmehr: 'versteht sie es, anstand mit heiterkeit $\mathrm{zu}$ vereinigen, so ist es, wie wenn lilie und rose anmutig gepaart sind'. Die lilie entspricht der zuht, die rose dem gemeit sin. Auch wol steht nicht in abgeblaßter bedeutung; dem ganzen ausdruck diu lilje stêt der rôsen b̂े kommt das prädikat wol zu. Die brachylogie sô stêt diu lilje für unser 'so ist es, wie wenn die lilie' usw. hat ihr genaues gegenstück bei Gottfried, Tristan $17983 \mathrm{ff}:$ swâ sô daz wîp ir wîpheit unde ir herze von ir leit und herzet sich mit manne, dâ honeget diu tanne, dâ balsemet der scherlinc; der nezzelen ursprinc der rôset ob der erden.

Auch die bestandteile des folgenden bildes $33-36 n \hat{u}$ merket wie der linden stê der vogele singen, dar under bluomen unde klê :noch baz stêt wîbe werder gruoz sind nicht nur so obenhin gewählt, wie bei Reinmar von Zweter an der von Wilmanns nach Simrock verglichenen stelle: milte und ellen haben nicht eben viel mit dem klee gemein; bei Walther entspricht dagegen der vogelgesang über dem blumenteppich sehr genau dem werden gruoz, der edlen conversation ${ }^{2}$ ) In den worten $n \hat{u}$ merket möchte ich nicht so sehr eine nachdrückliche ankündigung erblicken als ein syntaktisches mittel, über das die zierliche mhd. kunstsprache verfügt, wo wir schwerfälligere wendungen wie 'noch besser als der linde vogelgesang

1) Auch an den von Pfeiffer und Wilmanns citierten stellen stehen lilie und rose nicht rein formelhaft, am allerwenigsten an der ersten.

2) Rudolf von Fenis gibt mit schoener gruoz MF. 80, 22 rermutlich provenzalisches douz parlar wieder; s. Zs. fda. 55, 374. 
ansteht, steht frauen' usw. gebrauchen müssen. Ganz gleich gebaut sind die verse Wolframs, Lieder 5, 28. 29: selt waz ein storch den saten schade: noch minre schaden hânt mîn diu wîp.

Was den schluß des gedichtes betrifft, so stimme ich Braune darin vollkommen bei, daß ein wortspiel vorliegt, indem einen vaden 9 zuerst negation ist und dann eine andere bedeutung hat. Aber ich möchte doch den sinn der pointe etwas abweichend fassen. Ich sehe in den letzten worten der dame eine abfertigung des herrn, der am schluß seiner strophe, über das thema hinausgehend, etwas persönlich geworden ist: ir minneclîcher redender munt der machet daz man küssen muoz. Die dame geht scheinbar auf diesen ton ein. Wer die den frauen wohlgefälligen eigenschaften besitzt, der könne verlangen, wonach er begehrt. welch wîp verseit im einen vaden? - der herr muß glauben, daß dies bedeute: 'welche frau würde ihm auch nur das geringste abschlagen', und nun muß er hören, daß der faden wirklich nur ein faden ist: ja, seide kann er bekommen. Er begehrt natürlich etwas ganz anderes ${ }^{1}$ ).

46, 15. alsam der sunne gegen den sternen stât. Wilmanns vermißt in diesem bild die sinnliche anschaulichkeit. Walther vergleiche abstract den glanz der sonne und der sterne. Er sei vielleicht durch ein bild verleitet worden wie das Horazsche velut inter ignis luna minores. Wilmanns hat offenbar dem vers den sinn beigelegt: 'wie die sonne den sternen gegenüber steht'. Das wäre natürlich unanschaulich, da man sonne und sterne nicht zugleich sehen kann. Aber Walther gibt uns deutlich zu verstehen, daß er an die aufgehende sonne denkt. Wie vor dieser die sterne verbleichen, so vor der eintretenden dame alles andere. stât hat hier perfectiven sinn: 'der sonne gleich, die den sternen gegenüber tritt'.

46, $32 \mathrm{ff}$. Dieses lied ist nach meiner überzeugung bisher ganz unrichtig verstanden,worden. Der wahre sinn erschloB sich mir zwar in der hauptsache schon durch genaue betrachtung des gedichtes selbst, zum vollen verständnis gelang't man jedoch erst, wenn man es mit $49,25 \mathrm{ff}$. in beziehung setzt.

1) Wilmanns scheint ähnliche gedanken gehabt zu haben; wie er nun aber den schluß mit der 'humoristischen und zugleich höchst anschaulichen wendung' versteht, wird nicht klar. 
Da gilt es nun, über den begriff der liebe ins reine zu kommen. Mit Simrock-Wackernagels 'liebreiz' oder Pfeiffers 'anmut' ist gar nichts anzufangen. Wilmanns sagt zu 49, 34: 'liep bezeichnet das anmutige, angenehme, liebe die empfindung, die dadurch hervorgerufen wird; minne die liebe, insofern sie sich auf einen andern richtet'. Aber liebe muß sich doch immer auf einen andern richten. Die sache liegt vielmehr so. Allerdings ist liebe die empfindung, die durch etwas liebez hervorgerufen wird. Aber liep heißt nicht nur 'angenehm', sondern auch 'lieb, geliebt', und demgemäß kann liebe genau dasselbe bedeuten wie nhd. 'liebe'. Für diesen begriff hat das mhd. auch das alte wort minne. Aber während das unverbrauchte liebe sich nur auf die innere empfindung bezieht, hat sich an minne allerhand conventionelles beiwerk angesetzt: so kann man auch aus andern gründen minnen, als weil man verliebt ist. Walther stellt sich nun auf den standpunkt, daß es bei der minne nur auf die liebe, auf das persönliche liebesgefühl ankommen dürfe.

Das verkündet schon die erste strophe. Herzeliebez frowelîn, so setzt der dichter ein, und fährt in der 3. und 4. zeile der strophe fort: kund ich baz gedenken dîn, des hete ich willeclîchen muot. Diese verse sind nicht mit Wilmanns auf die 2. zeile got gebe dir hiute und iemer guot zu beziehen, sondern mit Pfeiffer auf die erste: herzeliebez frowelinn. Pfeiffer

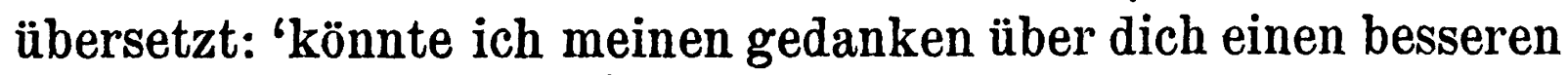
ausdruck geben (als herzeliebez)'. Richtiger werden wir den sinn so umschreiben: 'verstünde ich es, besser von dir zu sprechen, dich mehr zu preisen, d. $h$. ein besseres, lobenderes beiwort für dich zu finden als herzeliep, so wäre ich dazu gerne bereit. Aber was $k_{a n n}{ }^{1}$ ) ich denn noch mehr sagen, als da $B$ dir niemand holder, mehr zugetan ist'. holt und liep sind zusammengehörige begriffe. Wenn der dichter dem mädchen holt ist, so ist sie ihm liep; wenn ihr niemand holder ist, so ist sie niemandem lieber als ihm. Und das ist der höchste preis, den er ihr zu spenden vermag.

'Man tadelt mich' fährt Walther fort, ' $\mathrm{da} B$ sich meine dichtung an einen $\mathrm{zu}^{2}$ ) niedrigen gegenstand richtet. Ver-

1) Ich lese 49, 29 mit. Wackernagel nach $\Lambda$ waz mac ich nû sagen mo.

2) 49,82 lese ich mit $\mathrm{E}$ ze nider. 
wimscht seien die tadler, die keinen begriff von liebe, von der für die minne allein maßgebenden, vom stand unabhängigen, inneren liebesempfindung haben und ihr minnen von äußeren, objectiven werten, guot und schœne, abhängig machen. Mit diesen äußeren gütern ist keineswegs schon die liebe, auf die es allein ankommt, gegeben'. Bezüglich des guotes deutet dies der letzte vers der 4. strophe an: der dichter zieht den glasring der geliebten dem goldreif einer königin vor. Bezüglich der schœne sagt die 3. strophe ausdrücklich, daß sie sich mit $h a z$, dem gegenteil von liebe, paaren könne. Und dann gêt die schœene der liebe nâch, d. h. sie steht ihr nach und folgt ihr nach. Denn liebe macht ein weib schön ${ }^{1}$ ); die schönheit dagegen ist nicht imstande, ihrerseits ein wesen liep zu machen, und bekundet dadurch ihre minderwertigkeit.

Für Walther ist die geliebte schön und reich; daß sie es objectiv und für den kühlen beobachter nicht ist, ist ihm gleichgültig.

Aber das lied enthält nicht nur lehrhafte betrachtung, oder sagen wir lieber, es ist nicht nur gedankenlyrik. Walther hat es nicht nur mit seinen tadlern zu tun, sondern auch mit der geliebten. Banger zweifel drückt ihn, ob die geliebte ihm treu bleiben werde. Seine stimmung ist schmerzlich. owê, $d \hat{a}$ von ist mir vil $\left.w \hat{e}^{2}\right)$, so schließt die erste strophe, und mit owê danne, ob daz geschiht das ganze gedicht.

Wir lassen jetzt vorläufig dieses gedicht und wenden uns zu dem lied 46, $32 \mathrm{ff}$. Um seinen sinn zu erfassen, müssen wir von der zweiten strophe ausgèen. Hier gilt es aber vor allem, den richtigen text festzustellen.

Es ist klar, daß A und BCEF zwei familien sind, vgl. Wilmanns Zs. fda. 13, 223, Braune Beitr. 40, 216. BC gehören wieder enger zusammen. Die stellung von $\mathrm{F}$ innerhalb der familie ist nicht ganz sicher, was aber kaum von praktischer

1) Seltsam, daß J. Grimm, Grammatik 4, 484, Weinhold, Mhd. grámm. s. 565 und Wilmanns zu 50, 5 (liebe machet schone wîp) schœene für einen unflectierten acc. plur. halten. Es ist natürlich ebenso singular wie im folgenden vers lieben lîp. Dieselbe construction, artikelloses attributives adjectiv, wo wir prädicatives setzen würden, auch bei Hartmann MF. 205, 15: sît sinne machent soldehaften (BC: schadehaften) man.

2) So mit Wackernagel nach A. 
bedeutung ist. Die handschriften neigẹn dazu, die parallel gebauten verse der stollen im wortlaut möglichst anzugleichen. 47, 6 schreibt A nach 9 muot statt lîp. Umgekehrt BCE 8 haisset dú das (da B) nach 5 heizet diu sô. Und so ist auch 9 hoher wurde $\mathrm{A}$ das richtige und nicht das nach. Franker liebe 6 umgeformte werder liebe von BCF. Einen rest des echten hat noch E: Daz der muot so hohe stiget. Und endlich ist 7 liebe BCF zu verwerfen', schon deshalb, weil die unverwandten handschriften $\mathrm{A}$ und $\mathrm{E}$ minne überliefern. Im ganzen hat $\mathrm{A}$ den besseren text. Daher halte ich den wortlaut in der ausgabe von Wilmanns für richtig ${ }^{1}$ ). Die strophe lautet also:

47, 5 Nideriu minne heizet diu sô swachet daz der lîp nâch kranker liebe ringet:

diu minne tuot unlobelîche wê.

Hôhiu minne reizet unde machet

daz der muot nâch hôher wirde ûf swinget:

10 diu winket mir nû, daz ich mit ir gê.

Mich wundert wes diu mâze beitet.

kumet diu herzeliebe, ich bin iedoch verleitet:

mîn ougen hânt ein wîp ersehen, swie minneclich ir rede sî,

15 mir mac doch schade von ir geschehen.

Niedere minne und hohe minne werden in parallel gebauten sätzen nach ihren wirkungen definiert. Die niedere minne bewirkt, daß der leib nach kranker liebe trachtet, die hohe minne, daß der muot (wie ein edler falke) aufsteigt, um hôhe wirde zu erjagen. Zur niederen minne gehört also die liebe, zur hohen die wirde. Jene wird kranc genannt, diese hôch. Ebensowenig wie hôch ist kranc determinierendes adjectiv. Es gibt nicht etwa, wie BCF glauben, neben der kranken liebe auch eine werde; vielmehr gilt die eigenschaft liranc von dem begriff liebe in seinem ganzen umfang, so wie hôch von dem begriff wirde.

Da mithin liebe zur niederen minne gehört und stets kranc ist, kann die herzeliebe in zeile 12 unmöglich, wie man bisher angenommen liat, in den bereich der hohen minne fallen. lumet diu herzeliebe, ich bin iedoch verleitet heißt: wenn die

1) Fraglich ist nur, ob nicht 15 das wol von BCEF vorzuzichen ist. Fur den sinn ist das naturlich berlcutungslos. 
licbe sich zeigt, werde ich doch vom rechten weg abkommen, d. h. von dem weg, den das winken der hohen minne mir weist. Zugleich sehen wir, daß die definitionen des aufgesangs nicht aus der seele des dichters heraus gesprochen sind. Er gibt eine fremde meinung wieder, unter deren suggestion er steht. Ihm ist in wahrheit die liebe nicht kranc, das innige wort herzeliebe enthüllt seine wahre empfindung. Bloß unter dem einfluß einer fremden anschauung hat er rein reflexionsmäßig den wunsch, sich der hohen minne zuzuwenden; das bedeutet diu winket mir nî, daz ich mit ir gê. An einen bestimmten gegenstand der hohen minne denkt er nicht. Aber er weiB, $\mathrm{da} B$ er seinen entschluß nicht ausführen wird, wenn die herzeliebe sich zeigt. Und diese gefahr droht ihm von einer frau. Das weib z. 13 ist ein gegenstand der niederen minne. Sie kann ihm leicht schaden bringen, nämlich dadurch, daß sie die herzeliebe in ihm entzündet und ihn durch sie verleitet, von dem weg der hohen minne abführt.

Und nun verstehen wir auch die erste strophe. Der dichter ist im zustand qualvollen schwankens. Die niedere minne hat ihn fast getötet $\left.{ }^{1}\right), d . h$. nach der meinung der welt nichtswürdig gemacht, durch die hohe minne ist er siech geworden, d. h. seine empfindung sträubt sich gegen sie. Gibt es da keinen ausweg? In seiner bedrängnis wendet er sich an die Mâze: sie möge ihn ebene werben ${ }^{2}$ ) lehren. Denn wenn ihr widerpart, die Unmâze, die herrschaft der extreme, sich behauptet, so kommt er aus der not nicht heraus.

1) Wilmanns bemerkt zu ze nidere in dem vers 47,2 ich was vil nâch $z e$ nidere tôt: 'auf $\mathrm{zu}$ niedrige weise $\mathrm{d}$. $\mathrm{h}$. durch $\mathrm{zu}$ niedrige werbung'. Der zweite teil dieser erklärung trifft den sinn, der erste ist grammatisch unrichtig. ze nidere gehört nicht $\mathrm{zu}$ tôt, sondern bezieht sich prädikatähnlich auf $i c h$. Der satz enthält zwei mitteilungen: ich war ze nidere und dabei war ich tôt. Ebenso steht es mit hôhe in dem folgenden vers: nû bin ich aber ze hôhe siech. - Wegen der bedeutung von tôt vgl. 73, 16 .

2) ebene ist ganz wörtlich zu nehmen. Im sinne der Aristotelischen ethik, aus der ja in letzter linie der begriff der mâze stammt, betráchtet Walther das ebene als das $\mu \varepsilon ́ \sigma o v$ zwischen den beiden extremen des hohen und des niedrigen. Die umschreibungen 'im rechten ebenmaße der mitte' (Pfeiffer) and 'auf der mittelstraße' (Wilmanns) sind nicht genau. Gar nicht zu denken ist an richtiges mab innerhalb der hohen oder der niederen minne. Im sinne Walthers wäre es, neben der hohen und der niederen eine ebene minne anzunehmen. 
Wir werden der gequälten seele nicht vorhalten, daB sie unmögliches begehrt, da $B$ es nichts mittleres gibt zwischen hoher und niederer minne. Und Walther entwaffnet selbst den einwurf. Die Mâze stellt sich ja nicht ein - mich wundert wes diu mâze beitet - und der dichter bleibt der gewalt seiner neigung überlassen, die liebe siegt über die wirde.

Unser lied verhält sich $\mathrm{zu} 49,25 \mathrm{ff}$. wie in einem prozeB die duplik zur verteidigungsrede. Anklage und replik ${ }^{1}$ ) haben wir zu erschließen. Man hatte Walther vorgeworfen, daß seine dichtung einem zu niedrigen gegenstand gelte. Er antwortete darauf $49,25 \mathrm{ff}$., in der minne komme es nur auf die liebe, die empfindung des liebenden an, nicht auf äußere vorzüge der geliebten person. Seinen tadlern stellt er aus, dab sie sich durch solche äußere vorzüge, nämlich guot und schoene, bestimmen lassen. Die gegner erwiderten: 'die liebe, die du als leitstern deiner minne rühmst, ist etwas geringwertiges. Die wirkung deiner, der niederen minne ist, daß der leib, der schlechtere teil des menschen, nur nach dieser kranken liebe trachtet, daher würdigt die niedere minne ihren anhă̈nger herab. Und der schmerz, den auch diese minne bringt, vgl. 49, 30 owê, dâ ron ist mir vil wê - ist ruhmlos: diu minne tuot unlobelîche wê. Anders die hohe minne, der du vorwirfst, daß sie nur nach guot und schone tragt. Diese minne bewirkt, daß der muot, der edlere teil des menschen, nach hôher wirde strebt'.

Und Walther tut nun in dem lied 46,32ff. so, als ob er in seiner ansicht erschüttert wäre. Er gibt im aufgesang der zweiten strophe die meinung der gegner wieder, als ob sie seine eigene wäre, und er stellt sich 47,2 so, als glaubte er wirklich, daß er durch die niedere minne vil nâch tôt gewesen sei. Er ist scheinbar geneigt, dem wink der hohen minne zu folgen. Aber ihm ist nicht wohl dabei, er fühlt sich sicch. Und er weiß: wenn die verachtete, kranke liebe, die für ilm herzeliebe ist, an ihn herantritt, und schon schickt sie sich dazu an, dann wird die hohe minne umsonst winken, die liebe

1) Da ich nicht weiB, ob diese ausdrücke der osterreichischen rechtssprache allgemein ublich sind, bemerke ich, daB ich unter replik dio antwort des anklagers auf die verteidigungsrede verstehe, unter duplik dic nntwort des verteidiger auf die replik. 
behauptet das feld. Nüchtern ausgedrückt: Walther beharrt auf seinem standpunkt.

Wenn die gegner die von Walther so hochgepriesene liebe liranc nannten, so spielten sie mit der bedeutung des wortes. liebe heißt ja auch 'lust'. Die gegner schoben Walther die meinung unter, $\mathrm{da} B$ er die bloße lust über die wirde stelle. Damit rückt das lied noch in einen andern zusammenhang, der über Walthers eigene dichtung hinausreicht.

Die bedeutungen von liebe und minne standen einander nahe. Das war für die reflexionsfreudigen lyriker ein anla $B$, das verhältnis der beiden begriffe zu erörtern. Heinrich von Morungen tut das MF. 132, 19. Den wortlaut und sinn dieser strophe hat uns erst C. v. Kraus erschlossen: Zu den liedern Heinrichs von Morungen (Abhandl. der k. gesellsch. der wissensch. zu Göttingen, phil.-hist. klasse, NF. XVI, nr. 1) s. $25 \mathrm{ff}$. Ich setze den text nach seiner herstellung s. 27 hierher. Den sperrdruck wende ich an, um die richtige betonung anzudeuten.
Sît si herzen liebe heizent minne, son weiz ich wiech die minne heizen sol. minne wonet dicke in mînem sinne: hæt ich liebe, minne enbære ich wol. liebe diu gît mir hôhen muot, dar zuo fröud unde wünne. son weiz ich waz diu minne künne wan daz ich eine trûren muoz von ir.

Da die leute, meint Morungen, dem begriff herzen liebe den namen minne geben, so muß der begriff minne irgend einen andern namen bekommen, denn die beiden begriffe liebe und minne sind durchaus verschieden. Denn Morungen verbindet mit dem wort liebe die bedeutung 'freude'. Für diejenigen, von denen er in der ersten zeile spricht, waren dagegen liebe und minne synonyma: liebe bedeutete ihnen 'liebe'.

Man hat längst bemerkt, daß Walther 69, $1 \mathrm{ff}$. auf diese strophe Morungens anspielt. Morungen hatte die gänźliche verschiedenheit von minne und liebe = freude behauptet; Walther meint, minne ist minne, tuot si wol, d. h. wahre minne ist immer mit freude verbunden.

Aber auch Ulrich von Liechtenstein setzt in dem lied (XXVIII, Lachmann 429, 11 ff.), aus dem Wilmanns zu Walther 
49, 34 einige verse anführt, Morungens strophe voraus ${ }^{1}$ ). Er polemisiert gegen ihn, indem er die wesenseinheit von liebe und minne behauptet $(430,1 \mathrm{ff}$.$) : Statiu liebe heizet minne.$ liebe, minne, ist al ein: die kan ich in minem sinne niht gemachen wol zuo zwein. Dabei ist er aber nicht ganz gerecht gegen seinen gegner. Denn er nimmt liebe in der bedeutung 'liebe' und meint nur, daß eben beständige liebe freude gewähre. Vgl. namentlich 430,10-12: stoetiu liebe ist alsô guot, daz si stoete freude gît stoetem herzen alle zît. Diese verse gäben eine alberne tautologie, wenn hier liebe $=$ freude wäre.

Bei Morungen hängt die gegenüberstellung von minne. und liebe am wort; wenn liebe nicht doppelsinnig wäre, würde die strophe unmöglich sein. Walther hält sich an die sache, sowohl 69, $1 \mathrm{ff}$. wie 49, $25 \mathrm{ff}$. In diesem lied werden liebe und minne gar nicht in gegensatz gestellt, wohl aber wird dem begriff liebe ein schärfer umrissener inhalt gegeben. Walthers gegner, die ihm mit der kranken liebe kamen, klammerten sich wieder an die amphibolie des wortes ${ }^{2}$ ).

Anmerkung. Ich habe oben liebe als das unverbrauchte, minne als das alte wort bezeichnet. Ich möchte da nicht mißverstanden werden. liebe setzt ja ahd. liubî fort, das auch 'liebe' bedeuten und geradezu als synonym von minna gebraucht werden konnte: Otfrid V, 7, 3-5 Habeta si nu, in uuar min, minna mihilo sin, mihilo liubi, thes unortes mir giloubi, Minna mihilo ubaral. Vgl. auch V, 7, 37f. Aber in gewissem sinne ist liebe doch wiederum eine neuschöpfung, wie das ie statt des ahd. iu bezeugt. Die beziehung zu dem adjectiv liep ist eben immer frisch und lebendig geblieben und hat die bedeutung des substantivs bestimmt. minne dagegen war zum technischen wort der modernen höfischen lyrik erstarrt.

1) Den einfluß Morungens auf Ulrich betont v. Kraus, $\mathrm{Zu}$ den liedern Heinrichs von Morungen, s. 30 anm. 5.

$\left.{ }^{2}\right)$ Einen nachklang der erörterungen über liebe und minne findet man noch bei Kristan von Hamle (Bartsch, Liederdichter 32) und Konrad von Kilchberg (Bartsch 85). Kilchberg sagt 19-24 mit einer reminisceuz an Wolfram, Parzival 532, 11-18: $O b$ mich des ir güete, ir tugent note daz ich si sô herzeclîche minne, dâ ist Vênus gar ân alle schulde. Amor, ist dîn vackel heiz, selker noete ich niht enweiz. wâriu liebe ist minne ein ibergulde. Er hat liebe im sinne Walthers genommen. Nicht ganz klar ist mir die bedeutung von liebe in den versen Kristans von Hamle 27-32: Dâ brennet diu minne vor liebe als ein gluot, dannoch grozer counder diu minne dâ tuot. sie lât sich münde an ein ander vergezzen. aldi hât diu minne mit maneger fröide sorge unde trûren vil gar übersexzen, aldá hât diu liebe die minne aberwegen. 
50, 35-38. Swanne ichs alle schouwe, die mir suln von schulden wol behagen, sô bist duz min frouwe:

daz mac ich wol âne rüemen sagen.

Die übliche erklärung von zeile 37 ist im höchsten grade unbefriedigend. 'Wenn ich alle diejenigen betrachte, die mir 'von rechts wegen gefallen müssen, so' - man erwartet: 'bist du diejenige, die mir am besten gefällt' oder: 'diejenige, die mir allein oder ganz besonders gefällt', oder: 'so übertriffst du sie alle'. Statt dessen soll Walther sagen: 'so bist du meine herrin!' Pfeiffer erklärt freilich: 'du stehst hoch über allen, die ich sah und die mir gefielen'. Aber das kann doch mîn frouwe nicht heißen, wenn man wie Pfeiffer frouwe mit 'herrin, gebieterin' übersetzt.

Und dann zeile 38: 'das darf ich ohne zu prahlen sagen'. Wilmanns bemerkt: 'rüemen bedeutet im minnesang: sich mit erfolgen bei der geliebten brüsten. Ein solches rüemen würde schon in ausdrücken wie friundinne oder trût liegen; frouwe, sagt Walther, könne er sein mädchen wohl ohne ruom nennen'. Wir wollen die richtigkeit des letzten satzes vorläufig zugeben; aber warum in aller welt spricht Walther eine allgemeine wahrheit aus, die hier ganz außerhalb jedes zusammenhangs steht? 'V. 38', fährt Wilmanns fort, 'rechtfertigt, daß er in diesem verhältnis den ausdruck frouwe braucht'. Rechtfertigt? Wird der ausdruck frouwe für ein mädchen niederen standes dadurch gerechtfertigt, daß ein gewisser anderer ausdruck eine prahlerei in sich schlösse? Befand sich denn Walther in der zwangslage, gerade hier das mädchen entweder seine frouwe oder seine friundinne zu nennen, so daß er von zwei übeln das kleinere wählte?

Versuchen wirs einmal mit einer andern syntaktischen structur in zeile 37, nehmen wir mîn frouwe als apposition $\mathrm{zu} d u$ und lassen wir das in duz steckende $e z$ das eigentliche prädicat sein: 'so bist du, meine herrin, es', nämlich diu mir wol behaget. Das ginge eher, obwohl immerhin einiger guter wille dazu gehört, die ergänzung vorzunehmen. Aber mit 38 ist auch so nichts anzufangen. Nimmt man als object des rüemen den dichter, so paßt der satz wie die faust aufs auge; ergänzt man als object dich, so ist das nicht nur sprachlich bedenklich, sondern macht auch den satz unwahr. 
Wir müssen uns nach einer andern erklärung von frouwe umsehen. Das ahd. neben frewî vorkommende frouwî̀ würde mhd. frouwe ergeben - Williram 53, 5 schreiben auch mehrere handschriften urouue. Ich meine nun, $\mathrm{da} B$ an unserer stelle dieses frouwe vorliegt: 'wenn ich alle mustere, die anspruch darauf haben, mir zu gefallen, so bist du meine freude'. Ich denke, das gibt einen vortrefflichen sinn; im vordersatz wie im nachsatz erscheint der begriff des wol behagen, denn sein höchster grad ist die freude. Aber Walther spielt mit dem wort frouwe. Er setzt voraus, daß der hörer zunächst an die bedeutung 'herrin' denkt, und fährt nun fort: 'das darf ich ohne prahlerei sagen', d.h. es ist keine prahlerei, wenn ich dich meine freude nenne, wie es allerdings ein rüemen wäre, wenn ich dich als meine herrin bezeichnete. Denn gegen Wilmanns ist zu sagen, daß ein gewisses rïemen auch darin liegt, daß ein herr eine dame seine frouwe nennt; setzt dies doch voraus, daß sie seinen dienst angenommen hat. So weit ist aber Walther noch nicht; durch das ganze gedicht zieht sich ja der zweifel, ob er dem mädchen irgend etwas bedeute.

Es wird zunächst sehr. kühn erscheinen, daß ich Walthers wortschatz um ein wort bereichere, von dem unsere lexika nichts melden. Aber die gründe für das schweigen der wörterbticher lassen sich recht wohl erkennen. frouwe= freude kann nicht oft vorkommen, denn eine kunstsprache vermeidet homonyma, namentlich für begriffe, die so häufig erscheinen wie 'frau' und 'freude' bei unsern mhd. dichtern. Und dann haben unsere lexikographen an die möglichkeit der gleichung frouwe = frouwî gar nicht gedacht. Unter den stellen, die das Mhd. wörterbuch III $419 \mathrm{f}$. für die mittelalterliche etymologie von trouwe anführt, ist so manche, die erst dann sinnvoll wird, wenn frouwe sowohl 'frau' als 'freude' bedeutet. So der anfang der verse aus dem Marienlied: $d u$ heizes vrowe inde bis alsô. Ferner das erste citat aus der Frauenehre. Wenn man den zusammenhang berücksichtigt, so erscheint die betonung si sint sô guot für allez guot, die frouwen sint und fröude gebent ganz matt und sinnlos; man muß lesen: dic frouwen sint und fröude gebent. Und wenn man die verse $1069 \mathrm{ff}$., denen die zweite stelle (v. $1081 \mathrm{ff}$ ) entnommen ist, im zusammenhang liest, so wird man sehen, daß namentlich v. $1089 \mathrm{fr}$. im was ir 
vröuven ivol bekant, der vrouwen vrouwen namen vant bedeutend gewinnt, wenn man übersetzt: 'der für die frauen den namen 'freuden' ersann'.

Endlich ist frouwe $=$ frouv $\hat{\imath}$ in lesarten, textentstellungen und mißverstandenen stellen verborgen. In seiner abhandlung $\mathrm{Z} \mathfrak{u}$ den liedern Heinrichs von Morungen s. 15 weist C. v. Kraus auf mehrere fälle hin, wo fröuden statt frouwen in den text gehört. Er nimmt an, daß die mitteldeutsche vorlage frouweden schrieb. Vgl. auch s. 48 anm. 2, wo fröiden MF. 140, 21 aus trouwen über die mittelstufen vrouwen, vrouweden hergeleitet wird. An der stelle MF. 128, 30 bietet die überlieferte lesart einen anhaltspunkt für die annahme von ursprünglichem vrouweden, sonst kommt man auch mit vrouwen $=$ freuden aus und 140, 21 könnte man die zwischenstufe vrouweden ersparen. Endlich erwäge man folgende verse Reinmars MF. 176, $5 \mathrm{ff}$.:
Aller sælde ein sælic wîp, tuo mir sô daz mîn herze hôhe stê, obe ich ie dur dînen lîp wurde frô,
$10 \mathrm{daz}$ des iht an mir zergê.
ich was ie der dienest dîn:
sô bistuz diu fröide min.
sol ich iemer lieben tac
oder naht gesehen,

$15 \mathrm{daz}$ lâ, frouwe, an dir geschehèn.

Das verspaar 11. 12 ist doch seltsam. Man erwartet in v. 12 den correlativen begriff $\mathrm{zu}$ dienest $\mathrm{zu}$ finden; darauf weist sô sowie das (e)z, das ja durchaus nicht nach lust und laune dem prädicat voranlaufen $\operatorname{kann}^{1}$ ). Der correlative begriff $\mathrm{zu}$

1) Der ausdruck 'vorläufer des prädicats' führt irre. Genetisch ist $e z$ das eigentliche prädicat, das nomen eine apposition, die der sprechende anfügt, weil er fühlt, daß dem hörenden nicht klar sein muß, wer mit dem $e z$ gemeint ist. So scheinen auch Grimm, Grammatik 4, 222 und Paul, Mhd. grammatik $§ 328$ die grammatische structur aufzufassen. Immer muß aber die situation derart sein, daß die richtige deutung des e̊ $z$ wenigstens nahe liegt. Auch wir können unter dieser bedingung uns manchmal so ausdrücken. Wenn ich an die tür eines bekannten klopfe, kann ich auf seine frage 'wer ists?', in der voraussetzung, daß er meine stimme erkennt oder mich erwartet, antworten: 'ich bins' und dann zur sicherheit meinen namen hinzufügen. Wenn ich mich dagegen einem unbekannten nenne, kann ich unmöglich sagen: 'ich bins $X$ '. 
dienest ist aber frouwe, vgl. Morungen MF. 140, 29. 30 so ist siz doch diu frouwe mîn: ich binz der ir dienen sol. Ich vermute, daß Reinmar frouwe geschrieben und ein wortspiel beabsichtigt hat ${ }^{1}$ ).

54, $37 \mathrm{ff}$. Das handschriftenverhältnis ist hier nicht ganz einfach. Wilmanns hat Zs. fda. 13, 220 gezeigt, daß E und F auf eine quelle zurückgehen, machte aber 221, fußnote darauf aufmerksam, da $B$ F auch übereinstimmungen mit A zeigt. Er schlo $B$ daraus, da $B$ der quelle von $F$ correcturen beigeschrieben waren.

$F$ ist also ein mischtext, der zum größeren teil auf der quelle von $\mathrm{E}$, zum kleineren auf der quelle von A beruht.

Es fragt sich nur, ob $F$ überall contaminiert, oder in je einer strophe nur einer quelle folgt. In strophe I finden sich nur übereinstimmungen mit $\mathrm{E}$, in II und IV dagegen sowohl mit $\mathbf{E}$ wie mit A. Jedoch könnte in II die übereinstimmung mit A 55, 15 zufällig sein; es ist denkbar, daß F das in *EF stehende eine einfach aus unachtsamkeit ausgelassen hat. Dasselbe gilt für das fehlen der ersten zwei silben (owe C, ich wene $\mathrm{E}$ ) von 55,16 . Und umgekehrt könnte in IV das $\mathrm{zu}$ sammentreffen mit $\mathrm{E}$ (28 und: nu AC; 30 noch $\mathrm{A}$, iht $\mathrm{C}$ fehlt; 31 niht vor (en)mügest) dem zufall zugeschrieben werden.

Für eine nähere berührung von F mit $C$ kann nur IV 55, 26 geltend gemacht werden: Vil minneklichú minne C, Mynnigliche $\mathrm{F}$ : Gencedeclichiu minne A, Gnade riche minne $\mathrm{E}$. Aber F kann den fehler selbständig verschuldet haben; es setzt ja einfaches adjectiv an stelle von adjectiv und substantiv.

Auf jeden fall scheidet $F$ aus, wenn es gilt, das verhältnis der drei anderen handschriften festzustellen. DaB A weder mit $C$ noch mit $E$ näher verwandt ist, bedarf keiner besonderen erörterung. $\mathrm{Zu}$ erwägen ist nur, ob $\mathrm{C}$ und $\mathrm{E}$ auf eine mittelquelle zurückgehen.

1. $\mathrm{CE}: \mathrm{AF}$.

II 15 alterseine niht erwerben $\mathrm{C}$, niht erwerben eine $\mathrm{E}$ : niht erwerben $\mathrm{AF}$.

IV 28 twinge $\mathrm{CE}:$ twingest $\mathrm{AF}$.

29 versuoche wer $\mathrm{CE}$ : und sich wa ez (was F) AF.

2) Liegt auch bei Ulrich ron Liechtenstein, Frauenbuch $660,9: 8 i$ frowe ob al den freuden min ein wortspiel vor? 
2. $\mathrm{CE}: \mathrm{A}$.

III $2 t$ yesprechen CE : sprechen A.

IV 33 du vor dem vocativ CE : fehlt $A$.

3. CEF : A.

II 9 von dir (durch dich F) CEF vor: A wach verlorn.

13 solle $\mathrm{CEF}$ : soltu A.

16 du soltest $\mathrm{CEF}:$ ir soltent $\mathrm{A}$.

IV 27 warumbe CEF : owe wes A.

28 du twingest hie CEF : fehlt A.

30 schouven CEF : sehen A.

33 vor dir gestüende (bestuende $\mathrm{EF}$ ) CEF : dir wider stucnde $\mathrm{A}$.

In vier fällen hat A sicher einen fehler: 13. 16.28 (s. unter 1. und 3.). Umgekehrt bietet $24 \mathrm{~A}$ das richtige, aber $\mathrm{C}$ und $\mathrm{E}$ könnten unabhängig von einander denselben fehler gemacht haben. An den übrigen stellen ist die unrichtigkeit der einen oder der andern lesart nicht unmittelbar einleuchtend.

Wo CE oder CEF gegen A stehen, läßt sich eine nähere verwandtschaft von $\mathrm{C}$ und $\mathrm{E}$ nicht beweisen. W'ohl aber geht sie aus zwei unter 1. angemerkten übereinstimmungen hervor.

55, 15 haben Paul und Wilmanns mit recht die lesart AF in den text gesetzt; frô Minne, wie Lachmann, der aus CE eine aufnahm, wegen des metrums schreiben mußte, ist gegen Walthers gebrauch; vgl. Wilmanns z. st.

Und auch in 29 halte ich die lesart $A F$ für richtig. Die unpersönliche construction $w \hat{a}$ ez div widerstê ist viel origineller als das recht gewöhnliche wer div widerstê. Und bei näherem zusehen ist dieses wer dir widerstê auch recht lahm; wer der minne vielleicht widerstand leisten wird, kann ja gar nicht fraglich sein. Walther denkt schon an das bild von der diebin: 'drückst du hier, so drück auch dort, und sieh, wo du auf widerstand stößest, d. h. wo, an welcher stelle sich das schloß deinem versuch, es zu öffnen, widersetzt'.

Man wird am sichersten gehen, wenn man mit A schreibt und sich wâa ez dir widerstê., Möglich ist aber, daß das erste wort des verses von $C E$ richtig überliefert wird und daß, die zeile ursprünglich lautete: versuoche wá'z dir viderstê. ${ }^{*} \mathrm{CF}$ hätte dann $w a z=w \hat{a}^{\prime} z$ für das pronomen gehalten und das ganz unpassende neutrum durch wer ersetzt. In einer vorstufe von $\mathrm{A}$ wäre dagegen das richtig verstandene waz in $v a$ ez aufgelöst worden, was dann aus gründen des versmaßes die 
änderung von versuoche in und sich nach sich gezogen hätte. Die übereinstimmung von $\mathrm{F}$ (was) mit der hier angenommenen ursprünglichen schreibung waz wäre natürlich bloßer zufall.

Als wahrscheinlicher stammbaum ergibt sich:

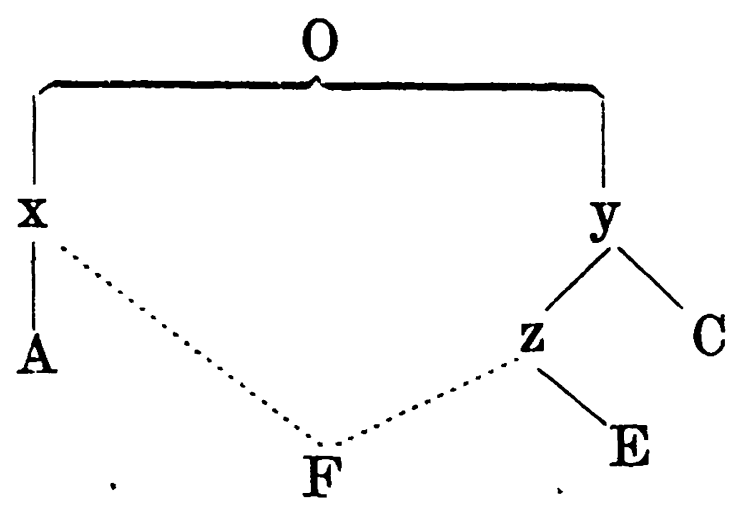

Jede übereinstimmende lesart von A und C sowie von A und $\mathrm{E}$ hat somit das präjudiz der richtigkeit. Daher setzte Wilmanns mit unrecht 33 die lesart von $\mathrm{E}$ in den text; der vocativ gehört an den schluß.

$A$ und $\mathrm{CE}$ sind zwei gleichberechtigte gruppen; nur innere gründe können zwischen ihnen entscheiden.

F würde nur dann eine stimme zukommen, wenn die früher geäußerte vermutung richtig sein sollte, daß diese handschrift innerhalb derselben strophe nicht contaminiert. Von praktischer bedeutung ist dies nur für einige verse der strophe IV, wo F ein $x$-text wäre. 27 würde die übereinstimmung mit $\mathrm{CE}$ war umbe gegen owe wes $\mathrm{A}$ sichern, ebenso 30 schouwen gegen sehen ${ }^{1}$ ) A, ferner 33 vor dir; freilich müßte man hier auch mit $\mathrm{EF}$ bestïende statt gestüende $\mathrm{C}$ schreiben. Immerhin könnte man aber in diesem vers $d a z e z$ in den text setzen; $e z$ ist in A überliefert, denn $e h$ ist klärlich daraus verlesen, der ausfall in $\mathrm{F}$ und y könnte ein zufälliges zusammentreffen sein.

34 gehen alle handschriften auseinander: tuon $\mathrm{A}$, rüne $\mathrm{E}$, slús $\mathrm{C}$, fehlt $\mathrm{F}$. Das richtige steckt offenbar in $\mathrm{E}$. Es ist nur fraglich, ob man mit Lexer 2, 1699 ein verbum $\hat{u} f$ rünen 'auftun' annehmen darf. Jedesfalls stand in der gemeinsamen vorlage aller handschriften und auch noch in $\mathbf{x}$ und $\mathbf{y}$ etwas, was dem rïne von $\mathrm{E}$ ähnlich sah. A hat, nur auf die nächste

1) Mir persönlich gefällt owe wes und sehen bessor (30 wltrde ich aber jedesfalls aus $C$ iht aufnehmen); jedoch beweisen laßt sich dio richtigkeit meiner empfindung nicht. 
hilfe bedacht, daraus das graphisch ähnliche tuon gemacht und die form als adhortativ verstanden; die änderung in tuo ist unstatthaft und ebenso unglaublich die meinung von Wilmanns, daß eine grobdialektische imperativform vorliege. C schrieb, da der sinn der stelle nicht zweifelhaft sein konnte, slus; $F$ lieb das unverständliche wort einfach aus: dem sinn genügte ja auch bloßes $u f$.

55, $35 \mathrm{ff}$. In dieser strophe, die gewiß nicht zu dem vorhergehenden gedicht gehört, gilt es vor allem, die stellung von B zu bestimmen, demnächst die stellung von $\mathrm{F}$.

$B$ zeigt berührungen mit $A, C$ und $E$; mit $F$ nur in fällen, wo noch eine dritte handschrift hinzutritt.

1. BA.

38 weichen $\mathrm{zwar}$ die beiden handschriften im wortlaut von einander $\mathrm{ab}$, stimmen aber darin überein, daß die zuhörer angeredet werden:

waz welt ir daz ich des nu tuo $\mathrm{A}$, nu ratent frunt was ich es tuo $\mathrm{B}$. Dem gegenüber steht in weis was ich dar vmbe tuo CEF.

2. BC.

a) 2 ane sehen $\mathrm{BC}$ : an gesehen $\mathrm{AEF}$.

3 ouge $\mathrm{BC}$ : ougen $\mathrm{AEF}$. nokel $\mathrm{BC}$ : nacke AEF.

b) 2 sine ruochet (si geruochet $\mathrm{B}$ ) mich niht ane sehen $\mathrm{BC}$; si wil m. n. an gesehen, $\mathrm{A}$; wie mac sie mich denne an $\mathrm{g} . \mathrm{\textrm {E }}$; wenn mag sie mich doch an $g$. F.

37 da enkan si niht erbarmen sich $\mathrm{BC}$; nu exwil si niht e. mich $\mathrm{A}$; du tanst auch niht e. dich $\mathrm{E}$; wen mag sie doch e. sich F.

c) BCF stehen zusammen in dem eben angeführten vers 37 , insofern sie sich haben gegen mich $\mathrm{A}$, dich $\mathrm{E}$. Ziemlich bedeutungslos ist in 3 der singular stuende, der in $\mathrm{BC}$ durch den singular ouge bedingt, in $\mathrm{F}$ wohl blober schreibfehler ist.

3. $\mathrm{BE}(\mathrm{F})$.

35 Dú salde $\mathrm{BE}$ : Vro selde $\mathrm{AC}$ (fehlt F).

mich BEF : sich AC.

Die übereinstimmung von BA v. 38 kann nicht zufällig sein. Sie geht entweder auf das original zurück oder stammt aus einer abgeleiteten quelle.

Dieselbe alternative gilt für einige $\mathrm{BC}$ : $d a$ enkan si niht erbarmen sich 37; sine ruochet (si geruochet) 2; noekel 3.

Kaum zufällig ist auch mich $35 \mathrm{BEF}$.

Man könnte nun zu einem einfachen stammbaum gelangen, wenn man annähme, daß $38 \mathrm{BA}$ insofern das richtige bieten, 
als auch das original die zuhörer anredete. Dann ergäbe sich sofort, daß CEF zu einer familie gehören.

Daraus würde weiter folgen, daß jede nicht fehlerhafte übereinstimmung von $B$ mit einer handschrift dieser familie das präjudiz für sich hätte. Man müßte mit Lachmann 2 sin ruochet in den text setzen und 37 in $d a$ enkan das relativ ursprünglichste anerkennen, aber auch 3 nokel schreiben. Das zusammentreffen von AEF in nacke wäre zufällig. Ebenso könnten AEF in 2 unabhängig von einander zu an gesehen gelangt und ane sehen das richtige sein. Umgekehrt wäre der singular in 3 (ouge - stuende) einem zufälligen zusammentreffen von $\mathrm{B}$ und $\mathrm{C}$ zuzuschreiben.

Dagegen würde aus 2 eine nähere verwandtschaft von $\mathrm{E}$ und $\mathrm{F}$ folgen, da beide handschriften hier einen fragesatz haben statt des richtigen sin ruochet, von dem si wil A eine leichte umformung ist.

Der stammbaum wäre demnach

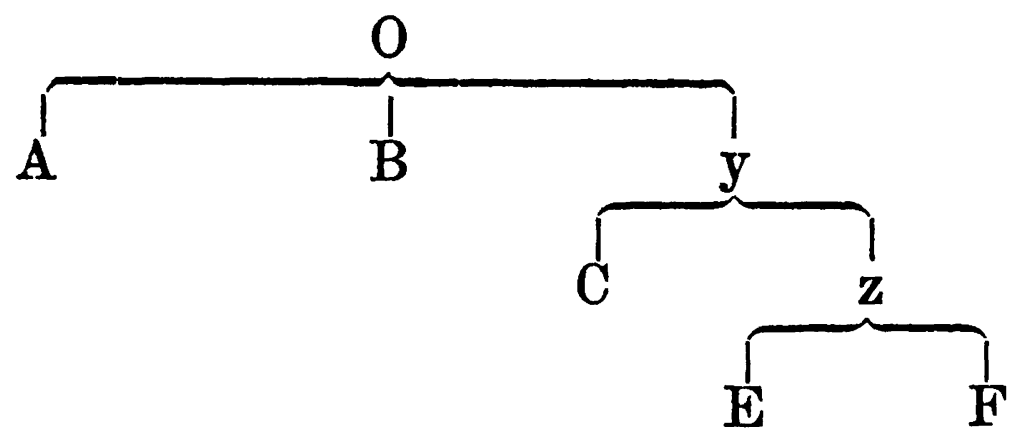

Eine kreuzung läge 35 vor zwischen $\mathrm{BE}(\mathrm{F})$ und $\mathrm{AC}$. Hier müßte auf einer seite der zufall gewaltet haben. Der stammbaum müßte deshalb noch nicht aufgegeben werden.

Aber dieser stammbaum enthält nur einen teil der wahrheit. Allerdings gehen im v. $38 \mathrm{CEF}$ auf eine abgeleitete quelle zurück, die geändert hat. Die anrede in $\mathrm{BA}$ ist viel lebhafter, origineller als das blassere in weiz waz ich dar umbe tuo von CEF. Die änderung von ${ }^{*} \mathrm{CEF}$ begreift sich leicht; die umbildung zur apostrophe liegt viel ferner. Aus denselben gründen fällt die entscheidung $z$ wischen $B$ und $A$ zugunsten von $B$ aus. Aber 37 steht $B C$ hinter $F$ zurück - $A$ und $E$, die die stelle umdichten, kommen gar nicht in betracht. Die lesart von $\mathrm{F}$ wen mag sic doch erbarmen sich ist ganz vortrefflich und keinem gewöhnlichen schreiber zuzutrauen. Man darf natürlich wen nicht als wenne auffassen; es ist wan 'warum 
nicht'. 'Warum erbarmt sie sich doch nicht' - doch in solchen wan-sätzen auch Iwein 3140. 5491. Diesem lebhaften ausruf gegenüber klingt $\mathrm{BC}$ schal. In der überlieferten form ist die lesart überhaupt nicht $\mathrm{zu}$ retten - was soll hier da? Und Lachmanns $j \hat{a}$ leuchtet nicht ein. da enkan geht auf wan kan zurück. Entweder durch bloße flüchtigkeit: dem schreiber klang die frage im ohr, und er setzte die antwort, daher das einleitende $d a$. Oder er hielt wan für die causalpartikel, stieß sich an der wortstellung und änderte mit möglichst geringer anstrengung und möglichst großer graphischer. anlehnung an die vorlage.

Nur insofern dürfte auch $\mathrm{F}$ vom ursprünglichen abgewichen sein, als, nach den übrigen handschriften zu schließen, das verbum kan und nicht mac war.

Bietet aber $37 \mathrm{~F}$ das richtige, so muß $\mathrm{B}$ mit $\mathrm{C}$ verwandt sein, und zwar näher verwandt als $C$ mit F. Früher fanden wir aber, daß im v. $38 \mathrm{~B}$ im gegensatz zu der ganzen familie $\mathrm{CEF}$ das echte überliefert. Der stammbaum ist zerstört. Entweder ist $\mathrm{B}$ ein mischtext oder $\mathrm{C}$.

Dieses ergebnis ist trotz der sonstigen engen beziehungen zwischen $B$ und $C$ nicht so verwunderlich. Denn unsere strophe steht nicht in der fortlaufenden reihe der töne, die in $B$ und $C$ in derselben folge erscheinen. Sie ist in B ein nachtrag.

Wenn Wilmanns s. 6 darin recht hat, daB schon *BC die strophe als nachtrag brachte, würde sich ergeben, daß es $\mathbf{C}$ war, der contaminierte, indem er v. 38 den text von ${ }^{*} \mathrm{EF}$ an die stelle von ${ }^{*} \mathrm{BC}$ setzte ${ }^{1}$ ).

Auf jeden fall haben BC nur éine stimme. Nur der hinzutritt einer sicher unverwandten handschrift, und das ist hier nur A, kann die richtigkeit einer lesart BC erhärten.

Ein solcher fall schien im eingang von v. 2 vorzuliegen. Als text von ${ }^{*} \mathrm{BC}$ ist anzunehmen sin ruochet mich niht ane sehen, als text von ${ }^{*} \mathrm{EF}$ wan mac si mich doch an gesehen. A stellt sich mit seinem si wil mich niht an gešehen, wie es

1) Ganz ähnlich liegen die dinge in den nachtragsstrophen B 85.86 $=51,5 ; 50,19$. Hier teilt $B$ mit $E$ eine reihe von fehlern, von denen $\mathrm{C}$ frei ist, vgl. 50, 25. 26; 51, 8. 12. Wenn $\mathrm{B}$ die lesarten von $* \mathrm{BC}$ wiedergibt, so hat $\mathrm{C}$ seine hauptquelle $\left({ }^{*} \mathrm{BC}\right)$ diesmal mit einem correcteren text verglichen. 
scheint, auf die seite von BC. Man müßte also annehmen, daß $* \mathrm{EF}$ nach dem muster von v. 37 auch in v. 2 den wansatz eingeführt hat, während der dichter deu ausdruck variierte. Das ist möglich.

Aber die übereinstimmung von $\mathrm{A}$ und $\mathrm{BC}$ wiegt doch nicht so schwer, wie es auf den ersten blick scheint. Ebenso wie in $F$ die verse 37 und 2 gleich gebaut sind, setzt $A$ in beiden zeilen dasselbe verbum finitum: nu enwil si niht erbarmen mich 37; si wil mich niht an gesehen 2. Daß deutet vielleicht darauf, daß $\mathrm{A}$ in seiner vorlage den gleichen bau der beiden verse vorfand. Die übereinstimmung von $A$ und $B C$ wäre also zufällig.

Mit sicherheit ist der fall nicht zu entscheiden. Ich möchte aber doch glauben, daß Walther auch v. 2 die lebhafte form des ausrufs gewählt hat. Sie paßt ausgezeichnet zu dem komisch aufgeregten ton der ganzen strophe. - Da 2 und 37 parallel gebaut sind, wird auch in 2 kan gestanden haben statt des von EF überlieferten mac.

Der stammbaum wäre also entweder

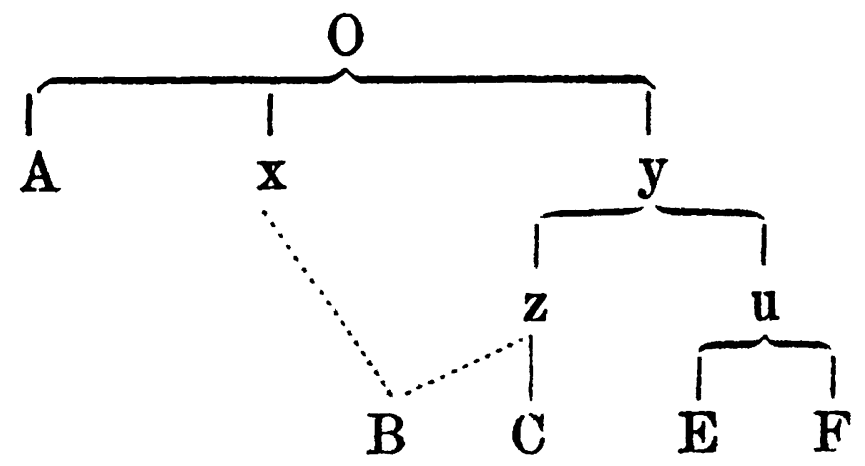

oder wahrscheinlicher

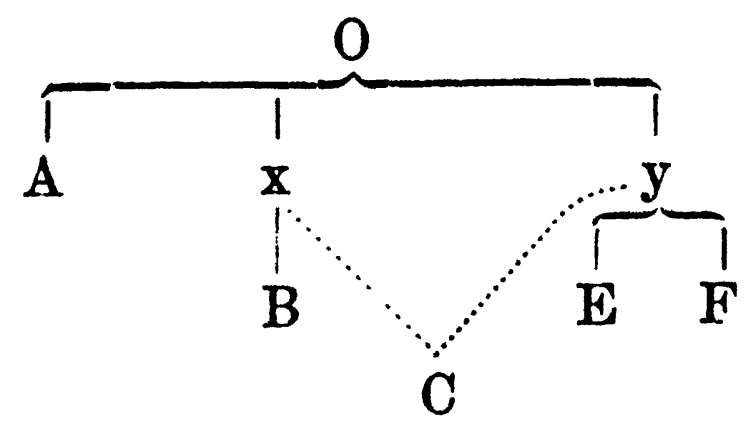

Die engere verwandtschaft von EF folgt im zweiten schema mit evidenz aus 38 ; im ersten läßt sich nur die ersetzung von kan durch mac 2 für sie geltend machen.

Es bleibt noch v. 35 zu erörtern, wo $\mathrm{BE}(\mathrm{F})$ und $\mathrm{AC}$ einander gegenuberstehen. 
Da v. 37 das reimwort sich ist, muß 35 das mich von $B E F$ das richtige sein; der rührende reim ist Walther nicht zuzutrauen. $\mathrm{A}$ und $\mathrm{C}$ haben unabhängig voneinander dafür sich eingesetzt, da die verbindung des reflexivs mit einer präposition etwas sehr gewöhnliches ist; hinder sich, vür sich sind ja später geradezu zu adverbien geworden. Wie nahe die änderung lag; zeigt $\mathrm{E}$, der zuerst sich schrieb und dann tilgte, als er merkte, daB er sich geirrt hatte. In A zog dann die einsetzung von sich die recht törichte umdichtung von 37 nach sich, während $\mathrm{C}$ sich um den von ihm geschaffenen rührenden reim nicht kümmerte.

$\mathrm{Ob}$ Vro selde $\mathrm{AC}$ oder Dú scelde $\mathrm{BE}$ richtig ist, läßt sich mit sicherheit nicht entscheiden, die wahrscheinlichkeit spricht aber für AC. B und $\mathrm{E}$ haben hier unabhängig voneinander geändert.

Der text der strophe wäre also:

Frô Sælde teilet umbe mich, und kêret mir den rugge zuo. wan kan si doch erbarmen sich! nû râtet, friunt, waz ich es tuo! si stêt ungerne gegen mir:

louf ich hin umbe, ich bin doch iemer hinder ir. wan kan si mich doch an gesehen! ich wolte daz ir ougen an ir nacke stüenden: sô müest ez ân ir danc geschehen.

58, 18. Alle herausgeber seit Lachmann schreiben anders diene ich swâ ich mac, gegen die überlieferung, die auf swaz (swas C, swes E) weist. Und swaz ist auch in den text $\mathrm{zu}$ setzen. 'Im übrigen diene ich euch, soviel ich kann'. 'Im übrigen': nämlich, abgesehen davon, daß ich keine hohen sprünge mache ${ }^{1}$ ). Und daran schließt der dichter die schalkhafte wendung, die das maß seiner kräfte (swaz ich mac) bestimmt: jeden siebenten tag (bzw. am sonntag) steht er der minne zur verfügung. $\mathrm{Zu} \backslash$ swâ ich mac passen die schlußverse ganz und gar nicht. Der dichter kann auch im scherz nicht sagen, daß er sich einen andern dienst suchen, trotzdem aber der alten herrin einmal in der woche dienen werde.

1) So hat auch Simrock (s. 106 der ersten auflage seiner übersetzung) das anders aufgefaßt, aber trotzdem an Lachmanns swâ festgehalten, das er freilich anders deutet als die späteren: 'Ich dien' ihr sonst, wo ichs vermag'. 
60, 3-5. dîu wilt sêre gâhen, und ist vil unnâhen daz ich dir noch sül versmâhen. Statt vil $\mathrm{AE}$ haben $\mathrm{BC}$ ouch; für dir $\mathrm{BC}$ setzen $\mathrm{AE}$ dich.

Ich halte dich für das richtige. Es paßt nicht gut, daß der dichter die überzeugung ausspricht, die zeit sei noch ferne, wo die welt auf ihn keinen wert legen werde; nach seiner darstellung tut die welt ja alles mögliche, um von ihm loszukommen. Und $20 \mathrm{f}$. sagt er ausdrücklich: ichn weiz wie dîn wille stê wider mich. Dagegen paßt dich vortrefflich. 'Wie sehr du dich auch bemühst, mir zu entgleiten, ich lasse dich dich nicht los. Denn die zeit ist noch ferne, wo ich auf dich keinen wert lege'.

Zeile 4 möchte ich doch oder joch schreiben, worauf ouch $\mathrm{BC}$ hindeutet.

62, $6 \mathrm{ff}$. Gegen die echtheit des gedichtes erheben sich bekanntlich bedenken. Der reim genam:spileman 63, 3.5 ist ohne seitenstück bei Walther, und getar:wâr (denn so ist sicher $\mathrm{zu}$ schreiben) 62, 32. 34 hat nur scheinbar eine parallele an gar:jâr 124, 22. 23. Denn nach Zwierzinas nachweis Zs. fda. 44, $1 \mathrm{ff}$. gab es auch eine form $g \hat{a} r$. Freilich reimt Walther viel öfter găr; aber ähnlich verhält sich Reinmar, vgl. Zwierzina s. 91). Zwei abnorme reime in einem gedicht anzuerkennen, ist mir unmöglich; ich halte strophe 3 und 4 für unecht. Dann mu $B$ aber auch strophe 2 fallen, denn sie ist mit 3 und 4 durch das eingangswort Frowe, mit 3 außerdem durch den reim -ar (dar:war 62, 21. 23, 'getar:wâr 62,32.34) verbunden. Auch zeigt strophe 2 eine sprachliche inconcinnität: Frowe, ir sît schoene und sît ouch wert: den zwein stêt wol genâde bî. Es wird also den adjectiven schœne und wert als drittes glied das substantiv genâde angereiht. Etwas ähnliches findet sich bei Walther nicht, so oft er auch mehrere begriffe durch ein zahlwort zusammenfaßt. Vgl. dagegen $8,14 \mathrm{ff}$. diu zwoi sint êre und varnde guot... daz dritte ist gotcs hulde, der zwcier ïbergulde und 8,27, anderseits die unserer stelle ganz ähnlich gebauten verse 35, 29. 30: küicne unde mille, und daz or dá zuo state sî, so ist er vil gar gelobt:den ewein stôt wol das dritte bî.

2) Die bemerkungen Plenios Beitr. 42, 257 anm. 2 uberzougen mich nicht. 
Gegen die echtheit der ersten strophe des tons lassen sich dagegen keine bedenken geltend machen. Auch hat sie gar keinen zusammenhang mit den folgenden. Um eine verbindung herzustellen, haben mehrere herausgeber die 3. strophe vor die 2. gesetzt. Dadurch wird aber der von ihrem dichter gewollte zusammenhang mit der 4. strophe zerstört. Denn das guot, das der dichter 62,35 begehrt, ist der reine lîp 62,37 , den er sich als belohnung für seinen gesang wünscht. Und endlich ist unfuoge 62, 8 ein viel $\mathrm{zu}$ starker ausdruck für die sprödigkeit einer dame.

Ich glaube also, daß strophe 1 Walther gehört, strophe 2-4 ein lied bilden, das ein anderer in Walthers ton gedichtet hat.

$62,14.15$ werden von Lachmann und Wilmanns syntaktisch verbunden: swie sanfte ichz alsô lâze sîn, daz und ouch mê vertrage ich doch dur etewaz. Wilmanns erklärt: 'Wie sanftmütig ich mich also zeige, so will ich dies und noch mehr aus einem gewissen grunde ertragen'. Ich vermisse einen beweis dafür, daß mhd. alsô in dem fortführenden sinn von nhd. also gebraucht wurde. Auch tritt der doch offenbar beabsichtigte gegensatz zwischen unsanfter 15 und sanfte 14 nicht hervor, wenn die beiden verse $z u$ verschiedenen satzgefügen gehören. Endlich hat der dichter von strophe 2-4 den sprachlich-metrischen bau seines vorbilds so aufgefaßt, daß der letzte vers der strophe durch einen starken einschnitt von dem vorhergehenden getrennt ist.

Wackernagel, dem Bartsch und Paul folgen, schrieb, indem er swie durch wie ersetzte: wie sanfte ichz alsô lâze sîn! Dadurch werden die von mir vorgebrachten bedenken beseitigt oder gemildert. Aber es bedarf gar keiner conjectur, wenn man nur in 13 mit C unsanfte schreibt. Dann schließt sich der swie-satz vortrefflich an und rundet die periode ab: hoet er die stat als ich si hân, bestüiende in danne ein zörnelîn, ez wurde unsanfte widertân, swie sanfte ichz alsô lâze sîn. "Hätte er sowie ich die gelegenheit sich zu rächen, so würde er, wenn ihn ein kleiner zorn anwandelte, unsanft reagieren, so sanft ich die sache auf sich beruhen lasse'. In gleicher lage benehmen sich der klôsenære und Walther entgegengesetzt: jener widertuot, dieser lât alsô sîn, jener benimmt sich unsanfte, dieser sanfte. 
62, 20-22. Der syntaktische bau dieser sätze, der sinn von 20 und der wortlaut von 21 scheinen mir noch nicht gefunden zu sein. Pfeiffers erklärung von 20: 'hoffnungen und wünsche würde ich (meinerseits) fahren lassen, aufgeben' bringt einen fremdartigen gedanken, den gedanken der entsagung, herein und befriedigt auch deshalb nicht, weil ledic 20 offenbar mit frî 19 sinnverwandt ist. Wilmanns wiederum ist mit seiner deutung 'wolde ich, nämlich an eurer stelle' zu modern. Um dem vers $21 \mathrm{zu}$ einem auftakt zu verhelfen, schrieb Wilmanns höveschent die mîne sinne dar. Metrisch gefälliger wäre Wackernagels und höveschent mîne sinne dar. Aber die von C. v. Kraus, Zs. fda. 44, 149 ff. festgestellten bedingungen für die setzung des und sind hier nicht gegeben: 'wenn nur mein geist dort den hof macht, kann ich nichts tun, wenn er euch meinen gesang widmet', ist sinnlos. Ob man nun aber den vers ohne auftakt läßt oder so wie Wilmanns oder wie Wackernagel liest,

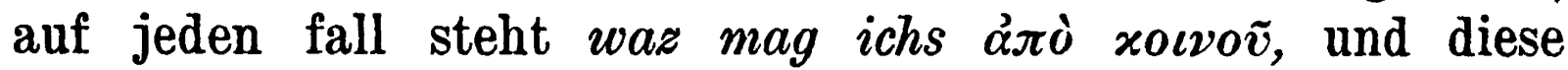
stilistische stümperei soll man auch einem unbekannten dichter nur dann zutrauen, wenn es nicht anders geht.

Alle anstöße schwinden, wenn man 21 syntaktisch von 22 abtrennt und näher an 20 rückt, indem man zugleich als auftaktsilbe $n \hat{u}$ (oder sô) vorsetzt. wân unde wunsch daz

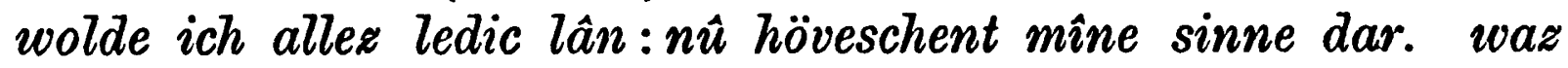
mag ichs, gebents iu minen sanc? 19 hieß es: joch sint iedoch gedanke frî, und nun fährt der dichter fort: 'ich möchte ja meine hoffnung und meinen wunsch herumwandern lassen; wo sie wollen, ich will sie ja gar nicht $z e$ iu bestoten (vgl. Reinmar MF. 181, 17). Nun ist es aber einmal so, dab meine sinne euch den hof machen. Was kann ich da tun, wenn sie meinen gesang euch widmen?' Der dichter stellt sich unschuldig, indem er sich und seine accidentien trennt (das scheint schon Pfeiffer erkannt zu haben): er selbst hat ja seinem hoffen und wünschen nicht das ziel vorgeschrieben, er selbst richtet ja seinen gesang gar nicht an die dame; an allem sind nur seine sinne schuld, sie machen der dame den hof, sie überreichen ihr das lied des dichters.

$73,13-16$. Wenn man in zeile 14, wie Lachmann und die andern herausgeber tun, mit AC só mac si wol verwanen sich schreibt, so schlottert der vorangehende satz swas si mir 
getuot wie ein schlechtsitzendes kleid. Denn 14-16 geben einen vollständig abgeschlossenen sinn, der keine erweiterung verträgt: 'Sie hat folgendes zu erwarten: wenn sie mich von meiner not befreit, so ist ihr leben durch mein leben geehrt; wenn sie mich dagegen tötet, so ist sie tot'. Was soll da der swaz-satz? Entweder enthält er eine schwächliche tautologie, indem er unbestimmt auf die alternative hinweist, die 15. 16 deutlich auseinandergesetzt wird, oder, wenn das mir getuot etwas anderes meint als nimet mich von dirre nôt und sterbet mich, ist der satz das reine geschwätz.

Es ist evident, daß $\mathrm{E}$ das richtige überliefert. dêst ein ende: swaz si mir getuot, des mac ouch si verwonen sich. 'Sie hat dasselbe für sich zu erwarten, was sie mir tut'.

WIEN. M. H. JELLINEK.

\section{ROLANDSTUDIEN.')}

\section{Rolandslied und Kaiserchronik.}

Seit Edward Schröders aufsatz über die heimat des deutschen Rolandsliedes (Zs. fda. 27, 70) und seiner einleitung zu der die 'scriptores, qui vernacula lingua usi sunt' der Monumenta

1) Ich citiere im folgenden Konrads gedicht nicht nach den an sich bequemeren und rationelleren fortlaufenden verszahlen von Bartsch (Leipzig 1874), sondern nach den seiten- und zeilenzahlen der ausgabe von Wilhelm Grimm (Göttingen 1838) und zwar aus einem bestimmten, für mich zwingenden grunde. Trotzdem Grimm lejder dies eine mal in seiner reichen herausgebertätigkeit den text nicht, wie er das sonst zu tun pflegt, nach moderner weise interpungiert und damit seine auffassung vom sinn und der verbindung der sätze eindeutig klargestellt, sondern den einförmigen versschließenden punkt aus der pfälzer handschrift beibehalten hat, ein mangel, dem-bei Bartsch abgeholfen ist, ist doch bei dem letzteren der sprachliche charakter der besten überlieferung durch eine willkürliche umsetzung in ein nicht einwandfreies rheinfränkisch, die auf weite strecken hinaus ohne jede handschriftliche gewähr dasteht, in einer weise verfälscht worden, daß man für den wortlaut im einzelnen unbedingt überall auf Grimms text :zurückgehen muB. Dazu will ich auch meine leser durch die citierweise nötigen und sie werden es nicht bereuen. Hoffentlich findet mein beispiel nachfolge: citieren wir doch uuch Freidank, Ulrich von Lichtenstein, Walther und so manchen andern text nach den seiten und zeilen der ersten kritischen ausgaben und Wolfram nach Lachmanns immerhin problematischen dreißigern, ohne vom modernen zählusus her daran anstoß zu nehmen. 